Supporting Information

\title{
A new adenine-derived physical dispersion system for graphene/polyimide composites
}

Zheng Lu, Zhengzhou Liu, Yang Liu, Weifeng Peng, Jianghuai Hu*, Gang Yang*

State Key Laboratory of Polymer Material Engineering, College of Science and Engineering, Sichuan University, Chengdu 610065, PR China 
Table S1. Basic characteristics of $\mathrm{rGO} / \mathrm{GO}$

\begin{tabular}{llllll}
\hline $\begin{array}{l}\text { Graphene } \\
\text { type }\end{array}$ & $\begin{array}{l}\text { Average } \\
\text { dimension } \\
(\mu \mathrm{m})^{\mathrm{a}}\end{array}$ & $\begin{array}{l}\text { lateral } \\
(\mathrm{x}\end{array}$ & $\begin{array}{l}\text { Average } \\
\text { dimension }(\mathrm{z})(\mathrm{nm})^{\mathrm{b}}\end{array}$ & $\begin{array}{l}\text { Oxygen content } \\
(\mathrm{wt} \%)^{\mathrm{c}}\end{array}$ & $\mathrm{I}_{\mathrm{D}} / \mathrm{I}_{\mathrm{G}}{ }^{\mathrm{d}}$ \\
\hline $\mathrm{rGO}$ & $\leq 10.00$ & 1.89 & 1.42 & 1.75 \\
$\mathrm{GO}$ & $\leq 7.00$ & 1.68 & 3.90 & 2.48 \\
\hline
\end{tabular}

${ }^{\text {a }}$ provided by the manufacturer

${ }^{\mathrm{b}}$ measured by atom force microscopy (AFM)

cmeasured by X-ray photoelectron spectroscopy (XPS)

${ }^{\mathrm{d}}$ measured by Raman spectroscopy

Table S2. Preparation recipe of OPI based nanocomposite films

\begin{tabular}{ccccccc}
\hline Sample & $\begin{array}{c}\text { ODA } \\
/ \mathrm{g}\end{array}$ & $\begin{array}{c}\text { ODPA } \\
/ \mathrm{g}\end{array}$ & $\begin{array}{c}\text { GO solution } \\
/ \mathrm{g}\end{array}$ & $\begin{array}{c}\mathrm{rGO} \text { solution } \\
/ \mathrm{g}\end{array}$ & $\begin{array}{c}\mathrm{NMP} \\
/ \mathrm{g}\end{array}$ & $\begin{array}{c}\text { Content } \\
/ \mathrm{wt} \%\end{array}$ \\
\hline 0.5-GO-OPI & & & 3.75 & - & 11.25 & 0.5 \\
1.0-GO-OPI & & & 7.5 & - & 7.5 & 1.0 \\
1.5-GO-OPI & & & 11.25 & - & 3.75 & 1.5 \\
2.0-GO-OPI & & & 15 & - & 0 & 2.0 \\
0.5-rGO-OPI & 0.5884 & 0.9116 & - & 3.75 & 11.25 & 0.5 \\
1.0-rGO-OPI & & & - & 7.5 & 7.5 & 1.0 \\
1.5-rGO-OPI & & & - & 11.25 & 3.75 & 1.5 \\
2.0-rGO-OPI & & & - & 15 & 0 & 2.0 \\
\hline
\end{tabular}

The study of interactions between artificial adenine derivative and graphene nanosheet

\section{Experimental}

\subsection{Dispersion of $r G O / G O$ in solvent assisted by diamine}

The polymerization solvent was determined by a simple process. To a $10 \mathrm{~mL}$ test tube, appropriate amount of $\mathrm{rGO} / \mathrm{GO}$ was added into two organic solvents (DMAc and NMP), at $0.5 \mathrm{mg} / \mathrm{mL}$ loading. The solutions were then subjected to ultrasonic treatment in an ultrasound bath cleaner for $2 \mathrm{~h}$. The treatment was carried out in 4 separate intervals to avoid an excessive heating of the mixture, and cool water after each interval should be replenished.

After that, an array of dispersion tests was conducted by varying the experimental variables ( $\mathrm{rGO} / \mathrm{GO}$ concentration, diamine concentration, centrifugation speed), the experiment was conducted as following (shown in Fig. S1). rGO/GO powder was added at a concentration of $2 \mathrm{mg} / \mathrm{mL}$ to a NMP $1 \mathrm{mg} / \mathrm{mL}$ solution of diamine ( $p$-APA/ODA). The six sets of mixture were then subjected to ultrasonic treatment that described above. The dispersions were then left to stand undisturbed after the ultrasonic treatment. Every other week, through mild centrifugation (2000 r, $20 \mathrm{~min}$ ) of the resulting dispersions afforded black-colored supernatants and estimated by using the Lambert-Beer law (measuring its optical absorption at $660 \mathrm{~nm}$ ). The constituent of samples was listed in Table S3, and be numbered from s-1 to s-6. 


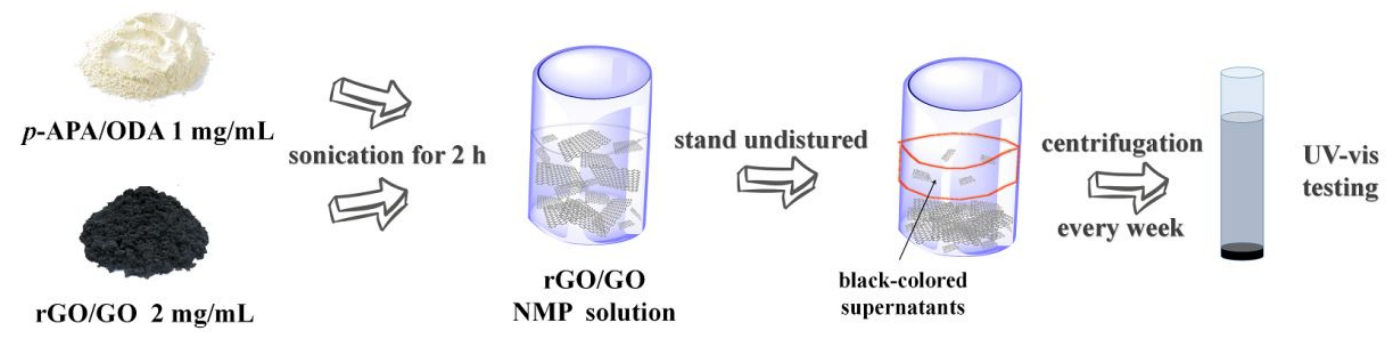

Figure S1. Dispersion experimental flow diagram

Table S3. Preparation recipe of testing samples

\begin{tabular}{|c|c|c|c|c|c|}
\hline sample & $\begin{array}{c}\mathrm{GO} \\
(2 \mathrm{mg} / \mathrm{mL}) \\
\end{array}$ & $\begin{array}{c}\mathrm{rGO} \\
(2 \mathrm{mg} / \mathrm{mL})\end{array}$ & $\begin{array}{c}p \text {-APA } \\
(1 \mathrm{mg} / \mathrm{mL})\end{array}$ & $\begin{array}{c}\text { ODA } \\
(1 \mathrm{mg} / \mathrm{mL})\end{array}$ & $\begin{array}{l}p \text {-APA-PA } \\
(3 \mathrm{mg} / \mathrm{mL})\end{array}$ \\
\hline S-1 & $\sqrt{ }$ & - & $\sqrt{ }$ & - & - \\
\hline S-2 & $\sqrt{ }$ & - & - & $\sqrt{ }$ & - \\
\hline S-3 & $\sqrt{ }$ & - & - & - & - \\
\hline S-4 & - & $\sqrt{ }$ & $\sqrt{ }$ & - & - \\
\hline S-5 & - & $\sqrt{ }$ & - & $\sqrt{ }$ & - \\
\hline S-6 & - & $\sqrt{ }$ & - & - & - \\
\hline S-7 & $\sqrt{ }$ & - & - & - & $\sqrt{ }$ \\
\hline S-8 & - & $\sqrt{ }$ & - & - & $\sqrt{ }$ \\
\hline
\end{tabular}

* $\sqrt{ }$ : the sample contained the substance at noted concentration; -: the sample didn't contain the substance

\subsection{The synthesis of model compound ( $p-A P A-P A)$}

As shown in Fig. S2, $40 \mathrm{~mL}$ of acetic acid was added into the $100 \mathrm{~mL}$ of round-bottom flask with $1 \mathrm{mmol}$ diamine ( $p$-APA). After the diamine was dissolved, $2.2 \mathrm{mmol} \mathrm{o}$-Phthalic anhydride was added and kept at $125^{\circ} \mathrm{C}$, refluxed for $5 \mathrm{~h}$. After cooling to room temperature, the resulting solid was gathered by suction filtration, and washed by using acetic acid and water until the filtrate was neutral. The precipitated yellow solid (namely $p$-APA-PA) was then refluxed for $5 \mathrm{~h}$ in methyl alcohol to get rid of residual acetic acid. After drying at $140{ }^{\circ} \mathrm{C}$ under reduced pressure for $8 \mathrm{~h}$, the titled product was obtained. ${ }^{1} \mathrm{H}-\mathrm{NMR}\left(400 \mathrm{MHz}, \mathrm{DMSO}-\mathrm{d}_{6}\right.$, TMS, rt) $\delta$ (ppm): 10.31 (s, 1H, N-H), 8.89 (s, 1H, C-H), 8.53 (s, 1H, C-H), 8.12-8.10 (d, 4H, Ar-H), 8.04-7.91 (m, 8H, Ar-H), 7.74-7.71 (d, 2H, Ar-H), 7.44-7.41 (d, 2H, Ar-H). (as shown in Fig. S3)

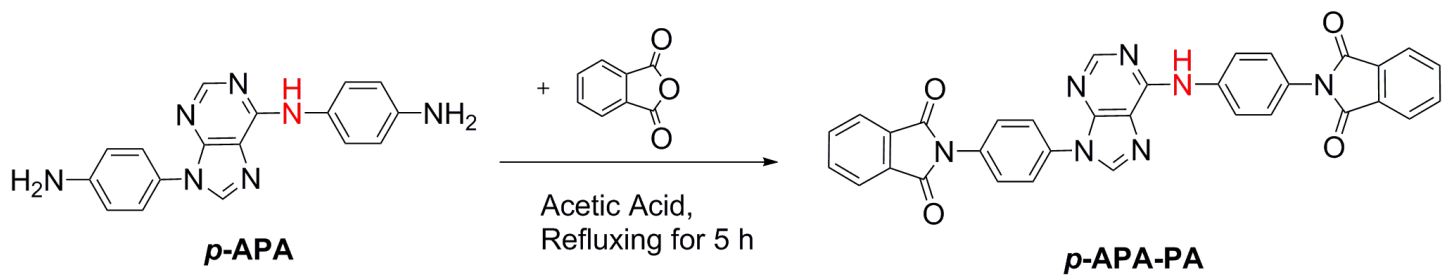

Figure S2. The synthetic procedure of model compound ( $p$-APA-PA) 


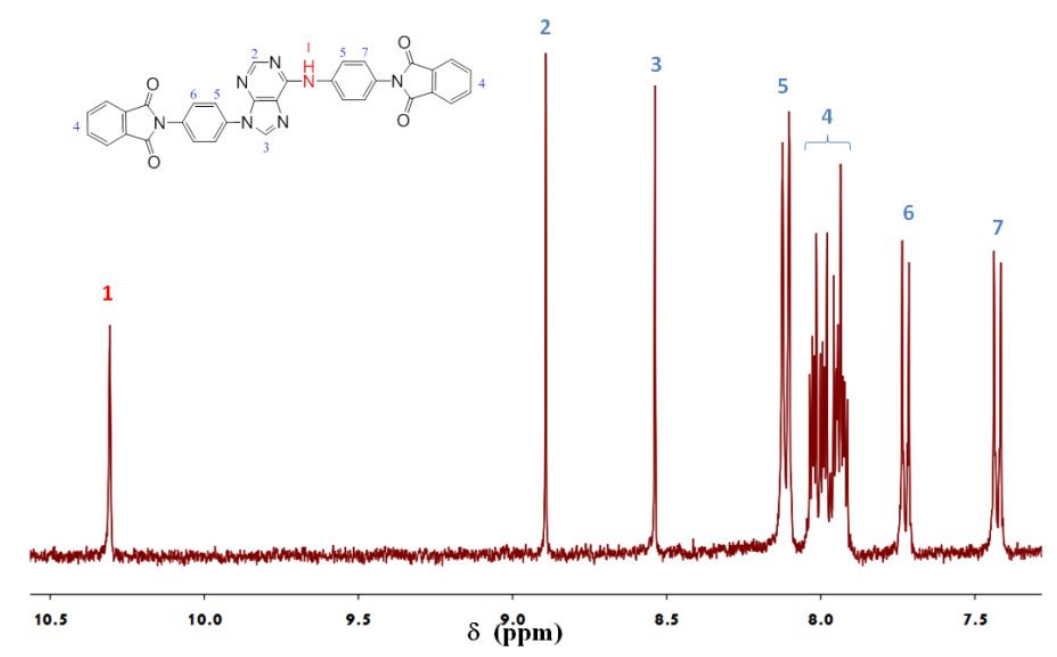

Figure S3. ${ }^{1} \mathrm{H}$ NMR spectra of model compound ( $p$-APA-PA)

\subsection{Absorption between adenine moiety and $r G O / G O$}

To study the interactions between graphene and polymer matrix by using model compound, following experiment was taken. The $\mathrm{rGO} / \mathrm{GO}$ powder was added at 2 $\mathrm{mg} / \mathrm{mL}$ into a prepared $3 \mathrm{mg} / \mathrm{mL}$ model compound ( $p$-APA-PA) NMP solution. The mixture was then subjected to ultrasonic treatment. The resulting dispersion was left to stand undisturbed for 2 weeks after the ultrasonic treatment, which will allow sufficient time for fully equilibrium of absorption. The constituent of samples was listed in Table $\mathbf{S 3}$ as well, and the samples were numbered as s-7 and s-8. To remove free, non-adsorbed model compound from the suspension, the undisturbed solution was subjected to a washing protocol via centrifugation (2000 r, $20 \mathrm{~min}$ ), and the top $70 \%$ of the supernatant was replaced by NMP and re-dispersion of the sedimentary mixture through a brief ultrasonic treatment. The sediment of $\mathrm{rGO} / \mathrm{GO}$ was dried in vacuum oven and measured by X-ray photoelectron spectroscopy (XPS) and Fourier transform infrared spectra (FTIR).

The interaction between $p$-APA and $\mathrm{rGO} / \mathrm{GO}$ was further studied by using UV-vis technique. The $\mathrm{rGO} / \mathrm{GO}$ powder was added at calculated concentration to a $0.03 \mathrm{mg} / \mathrm{mL}$ NMP solution of $p$-APA. The mixture was then subjected to ultrasonic treatment as well. The concentration of $\mathrm{rGO} / \mathrm{GO}$ was various at different level, 0.004 and $0.008 \mathrm{mg} / \mathrm{mL}$, respectively.

\subsection{The calculation of interaction energy between adenine moiety and graphene}

In order to study the interfacial bonding interaction of $\mathrm{rGO} / p$-APA mixed solution, the interface region of graphene/model compound (M) system was simulated. And the interaction energy $\left(\mathrm{E}_{\mathrm{int}}\right)$ can be calculated by the following equation [1],

$$
\mathrm{E}_{\text {int }}=\mathrm{E}_{\text {total }}-\left(\mathrm{E}_{\mathrm{M} \text { solution }}+\mathrm{E}_{\text {graphene }}\right)
$$

where $\mathrm{E}_{\mathrm{M} \text { solution }}$ is the potential energy of $\mathrm{M}$ ( $p$-APA-M or diphenyl ether) NMP solution, and $\mathrm{E}_{\text {graphene }}$ is the potential energy of graphene, $\mathrm{E}_{\text {total }}$ is the general potential energy of graphene $/ p$-APA mixed solution system. 
After the equilibrated $\mathrm{M}$ molecular was obtained, four main steps can be taken to calculate the $E_{\text {int }}$ between every $M$ system and graphene:

Step 1: To get the suitable model of graphene, geometry optimization (accuracy: fine, less than 500 steps) was performed under COMPASSII force fields. After that, a supercell of graphene was created with a $0.1 \AA$ vacuum slab which can change the periodicity from two-dimensional to three-dimensional. The system size was enlarged to $(25.56,14.75,0.1)$. This is nominated as the layer model-1.

Step 2: To build the layer model of $M$ solution, a few steps should be taken. NMP and M molecules were performed with geometry optimization (accuracy: fine, less than 500 steps) under COMPASSII force fields. Then, a cell with 8 NMP molecules and $2 \mathrm{M}$ molecules was constructed as layer model-2, the direction length of $\mathrm{x}$ and $\mathrm{y}$ should be changed (the same as the graphene surfaces). In order to reach an equilibrium state, 5 cycles of NVT anneal simulation from $300 \mathrm{~K}$ to $800 \mathrm{~K}(50 \mathrm{ps})$ was performed to loose the molecular systems firstly. Then, 500 ps MD-NVT simulation with a time step of $1 \mathrm{fs}$ ( $300 \mathrm{~K}$, accuracy: medium) was performed followed by $20 \mathrm{ps}$ MD-NPT simulation with a time step of $1 \mathrm{fs}(300 \mathrm{~K}, 1 \mathrm{~atm})$.

Step 3: To set up a mixed solution system, the graphene surface and the M NMP systems were taken as the first and second layer, respectively. To study the strength of $\mathrm{E}_{\text {int }}$ at different situation, the the second layer's vacuum thickness was set to $30 \AA$, and the vacuum thickness of the first layer was set to 3, 6, $9 \AA$, respectively. To reach an equilibrium state, 10 cycles of NVT anneal simulation from $300 \mathrm{~K}$ to $800 \mathrm{~K}$ with a time step of $1 \mathrm{fs}(100 \mathrm{ps})$ was performed. The atoms of graphene in these anneal simulations were fixed.

Step 4: The coordinates of graphene atoms were changed into unfixed after the graphene/M models were equilibrated. The energy of graphene/M NMP system, M solution and graphene was simulated by energy task (accuracy: ultra-fine). Then, according to Eq. (1-1), the $\mathrm{E}_{\text {int }}$ between graphene and M NMP solution was obtained.

The above-mentioned simulation was performed using Forcite and Amorphous module of MS 8.0.

\section{Results and discussion}

\subsection{The dispersion stability of $r G O / G O$}

In light of the research result that RNA/DNA nucleotides combined with proper nucleobases can be an effective stabilizer in graphene solution [2,3]. Therefore, we hypothesized that the artificial derivative of the nucleobase $p$-APA, a diamine monomer for polyimide, may act as an efficient graphene dispersant. On account of its unique aromatic heterocyclic structure, $p$-APA could be more efficient stabilizers of the nanosheets than the commonly used diamine (such as ODA), because of more strongly adsorption onto graphene. The Lambert-Beer law was used to calculate the concentration of suspension that obtained from the absorbance in UV-vis measurement. As shown in Fig. S4, we compared the dispersing ability of $\mathrm{rGO} / \mathrm{GO}$ sheet based on different diamine, $p$-APA and ODA. Fig. S4a showed the temporal evolution of concentration of GO suspensions in NMP solution containing $1 \mathrm{mg} / \mathrm{mL}$ p-APA (s-1), $1 \mathrm{mg} / \mathrm{mL}$ ODA (s-2), and without diamine (s-3). Similarly, Fig. S4b 
showed the result of rGO suspensions, for samples s-4, s-5 and s-6. It can be observed that the dispersion assisted by $p$-APA $(0.288 \mathrm{mg} / \mathrm{mL}$ for s- 1 and $0.154 \mathrm{mg} / \mathrm{mL}$ for s- 4$)$ exhibited much higher initial GO/rGO concentration than that of others $(0.125 \mathrm{mg} / \mathrm{mL}$ for $\mathrm{s}-2,0.095 \mathrm{mg} / \mathrm{mL}$ for $\mathrm{s}-3,0.100 \mathrm{mg} / \mathrm{mL}$ for $\mathrm{s}-5$ and $0.066 \mathrm{mg} / \mathrm{mL}$ for $\mathrm{s}-6$ ). Significantly, the results can be observed from Fig. S4a and b that except those obtained from the $p$-APA containing dispersion samples, other suspensions tended to precipitate mostly after standing for 5 weeks. After 5 weeks, the amount of the remaining GO and $\mathrm{rGO}$ in the s-1 and s- 4 was, respectively, $\sim 17.8 \%$ and $13.5 \%$ of their corresponding initial concentrations. And these values were reduced to below $6 \%$ for all the other samples which can be observed almost colorless. As can be confirmed by the digital photographs taken for those samples 5 weeks after their preparation (fig. S4c and d). There was virtually no suspended GO or rGO sheet in any solutions other than those of $p$-APA containing dispersion samples (s-1 and s-4). The enhanced solution stability of GO and rGO using adenine-based diamine reported here is assumed to be a result of the relatively strong interaction by $\pi-\pi$ stacking and hydrogen bonding interactions due to unique aromatic heterocyclic structure and extra secondary amine group (-NH-) of adenine. Base on the previously theoretical studies [4], GO complexes are stabilized by hydrogen bonding interactions between GO and bases, whereas graphene complexes are stabilized by $\pi-\pi$ interactions. And the bases are inclined to orient entirely parallel to the surface of the graphene relative to GO.
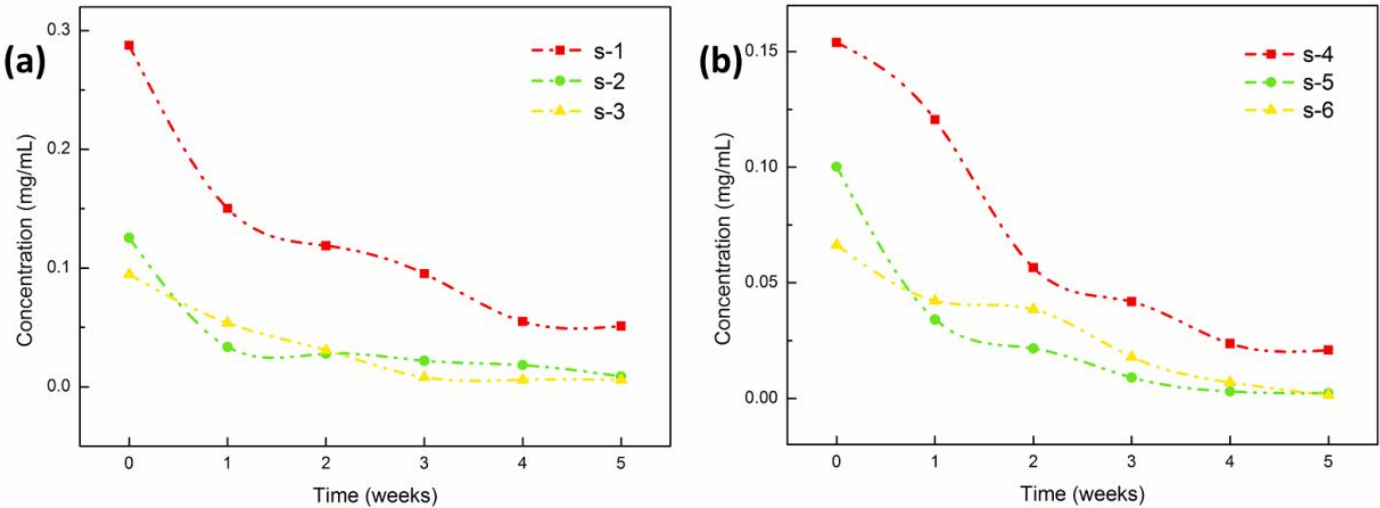

(c)

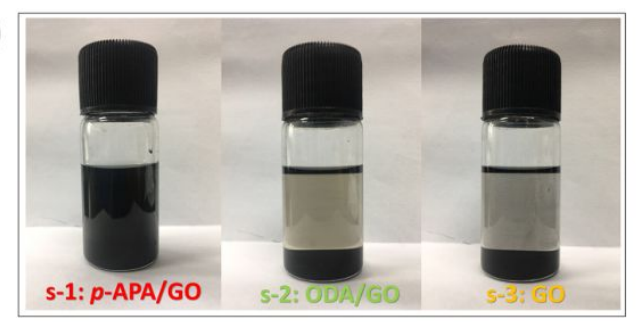

(d)

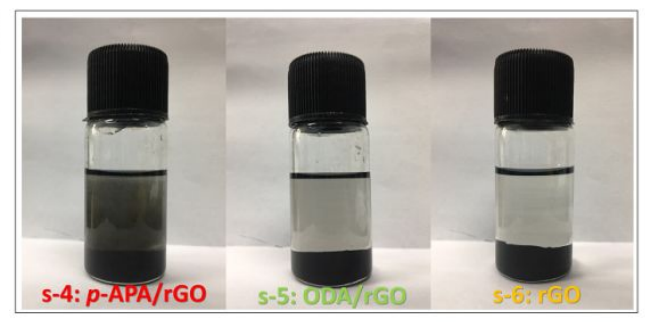

(e)
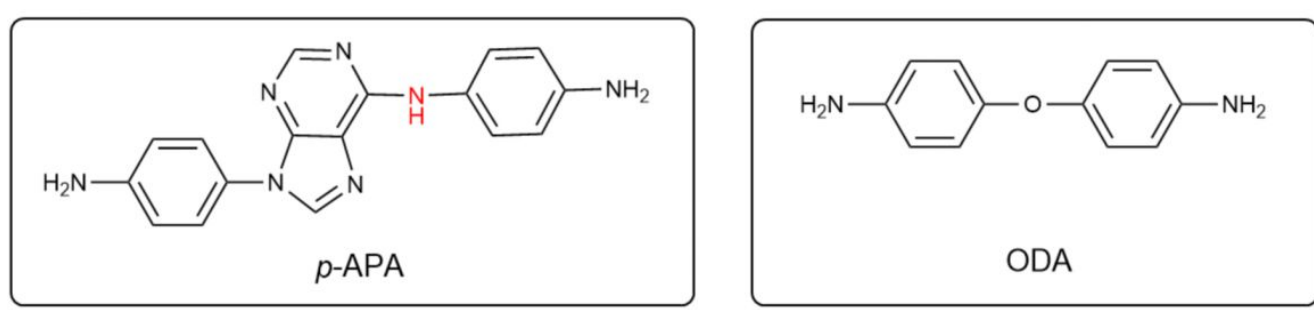

Figure S4. The stability of rGO/GO dispersions prepared with/without diamine: (a) sample s-1, 
s-2 and s-3; (b) sample s-4, s-5 and s-6; (c), (d) digital photographs of the dispersions taken 5 weeks after their preparation; (e) chemical structure of $p$-APA and ODA (Data in Fig. 1a and b was calculated by the data in UV-vis spectra using the Lambert-Beer law)

\subsection{Physical interactions between adenine moiety and $r G O / G O$}

For further demonstrating the non-covalent interactions between adenine moiety and $\mathrm{rGO} / \mathrm{GO}$ nanosheets, a model compound ( $p$-APA-PA) that has the similar repeat unit of the main chain of API was designed. The model compound was found to be easily absorbed on the surface of $\mathrm{rGO} / \mathrm{GO}$ sheet after standing undisturbed for 2 weeks resulting in co-precipitates of $p$-APA-PA/rGO or GO. The free $p$-APA-PA compound was removed by washing protocol as mentioned in experimental section to eliminate interference. The constitute of the co-precipitates (s-7 and s-8) was analyzed by FTIR and XPS as illustrated in Fig. S5.

As shown in Fig. S5a, the characteristic absorption bands of the imide group were observed around $1719,1374,716 \mathrm{~cm}^{-1}$ in $p$-APA-PA FTIR spectra, which were attributed to imide carbonyl asymmetric $\mathrm{C}=\mathrm{O}$ stretch, $\mathrm{C}=\mathrm{N}$ stretch and imide carbonyl $\mathrm{C}=\mathrm{O}$ bending [5]. In addition, there was no apparent absorption peak presented around the position of characteristic absorption of $p$-APA-PA in rGO and GO spectra, which brightened the comparison between the co-precipitates and pure model compound. Noteworthy from the FTIR spectra of s-7 and s-8, the absorption peak around $1714,1381,718 \mathrm{~cm}^{-1}$ as well as $1716,1382,716 \mathrm{~cm}^{-1}$ were corresponding to the characteristic absorption of $p$-APA-PA, indicating the actual existence of $p$-APA-PA in the co-precipitates after washing protocol. Besides, the relatively stronger intensity of the characteristic absorption of s-7 than that of s-8 inferred a higher content of $p$-APA-PA was absorbed on the surface of GO sheets.

As supplements, XPS analysis was performed to determine the elemental composition of the co-precipitates of s-7 and s-8 (Fig. S5b and c). The pristine rGO sheet was observed to contain $98.58 \%$ carbon and $1.42 \%$ oxygen. Similarly, the pristine GO sheet was observed to contain $94.9 \%$ carbon and $3.90 \%$ oxygen with trace amounts of sulfur which was likely merely contaminants [6]. One can notice from the XPS results of the co-precipitates of s-7 and s- 8 that there was an distinct increase in the content of nitrogen, up to $1.61 \%$ and $1.57 \%$. The variation in the elementary composition of the co-precipitates once again evidenced the existence of strong non-covalent bonding interaction between model compound and $\mathrm{GO} / \mathrm{rGO}$ sheet.

In addition, monitoring the shifting of absorption peak $\left(\lambda_{\max }\right)$ in UV-vis spectra is an effect experimental technique for further research of noncovalent $\pi-\pi$ stacking between planar molecule and graphene [7]. The segmental UV-vis spectra of $p$-APA solution with different concentration of $\mathrm{rGO} / \mathrm{GO}(0.004$ and $0.008 \mathrm{mg} / \mathrm{mL})$ were shown in Fig. S6. The spectrum of pure $p$-APA exhibited a $\lambda_{\max }$ at $314.5 \mathrm{~nm}$, which can be assigned to $n-\pi^{*}$ transition [8]. After the $\pi-\pi$ interactions with $\mathrm{rGO} / \mathrm{GO}$, the $\lambda_{\max }$ of $p$-APA was bathochromically shifted. The new peaks appeared at 316.5 and $317 \mathrm{~nm}$ for $\mathrm{rGO}$ at the concentration of 0.004 and $0.008 \mathrm{mg} / \mathrm{mL}$, respectively. And the new peaks appeared both at $316 \mathrm{~nm}$ for GO at the concentration of 0.004 and 0.008 
$\mathrm{mg} / \mathrm{mL}$, which confirmed successful $\pi-\pi$ interactions between $\mathrm{rGO} / \mathrm{GO}$ and $p$-APA. Since the rGO possessed a more flat basal surface than GO, a more distinct bathochromically shift can be observed at higher concentration of rGO, which suggested a stronger $\pi-\pi$ interactions.
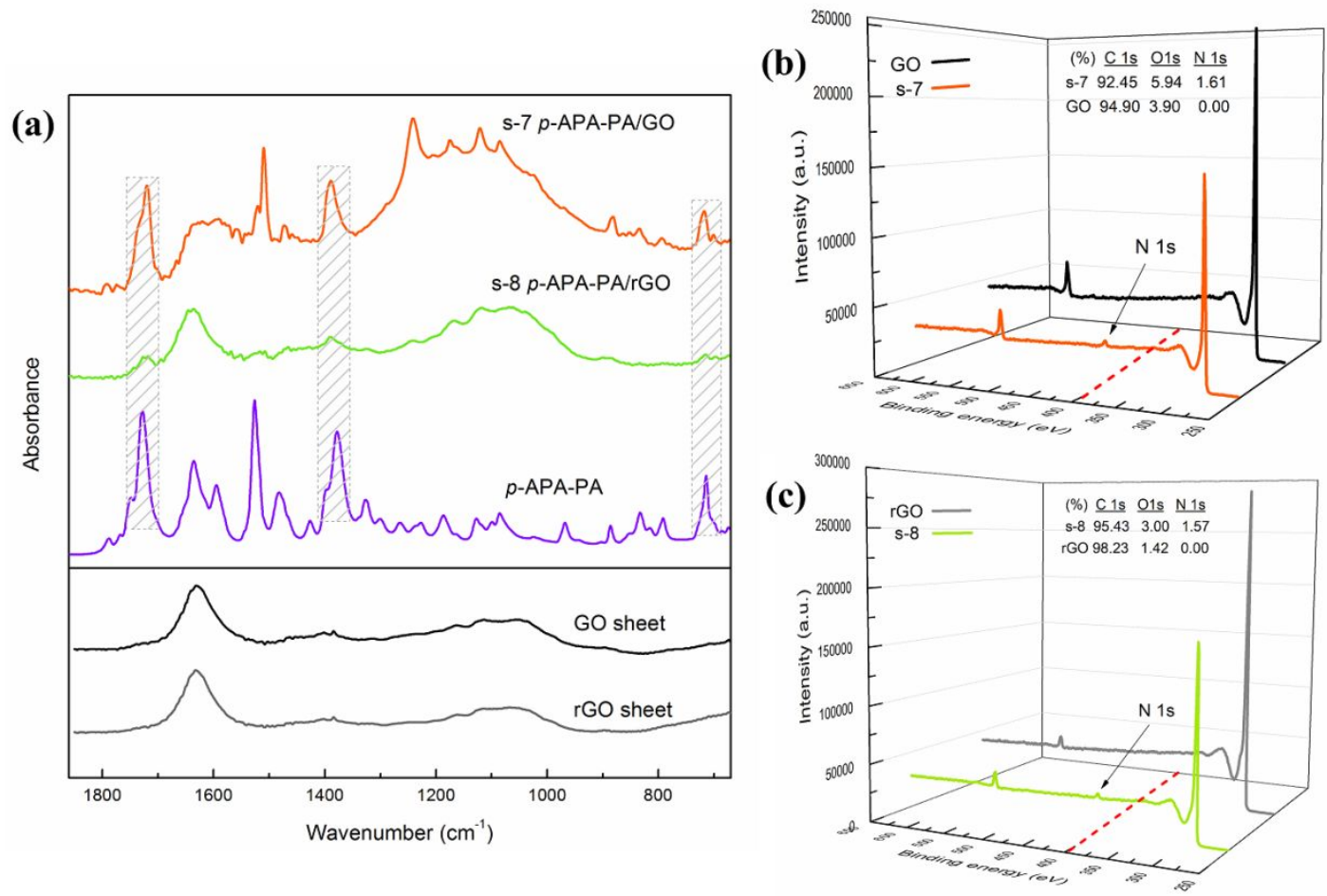

Figure S5. Characterization of $\mathrm{rGO} / \mathrm{GO}$ co-precipitates: (a) the FTIR spectra of $\mathrm{rGO}, \mathrm{GO}$, $p$-APA-PA and sediment of sample s-7 and s-8; (b), (c) the XPS spectrum of rGO, GO and sediment of sample s-7 and s-8

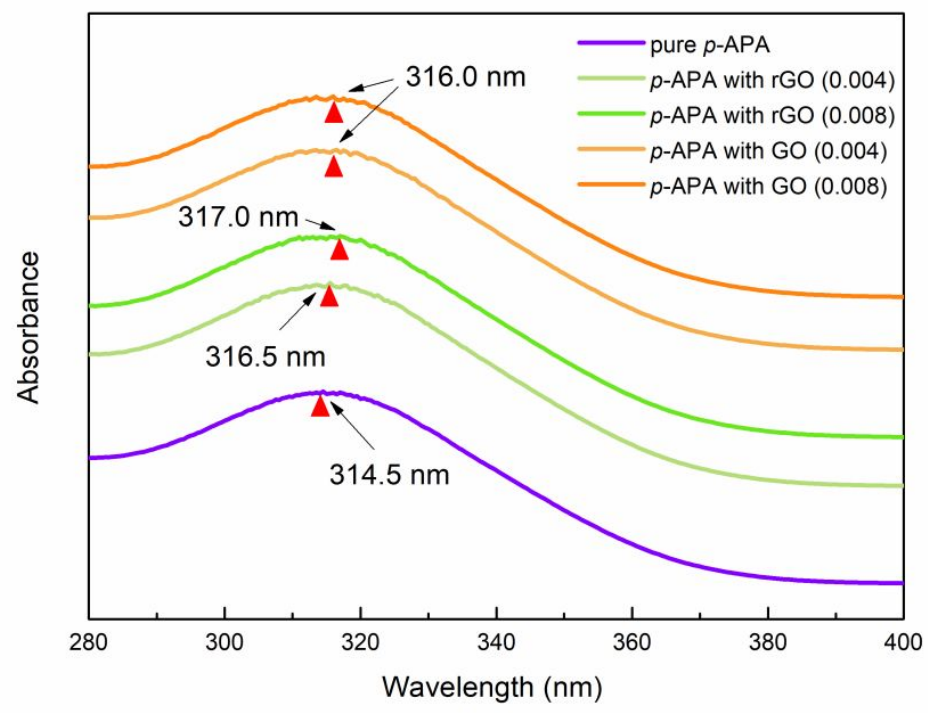

Figure S6. UV-vis absorption spectra of pure $p$-APA and $p$-APA with different loading of $\mathrm{rGO} / \mathrm{GO}(0.004$ and $0.008 \mathrm{mg} / \mathrm{mL})$

\subsection{Interaction energy by molecular simulation}


The dispersion stability of $\mathrm{rGO} / \mathrm{GO}$ solution could be improved by introducing adenine derivative, and the degree of improvement is directly related to the $E_{\text {int }}$ between compound and graphene [9]. Although the constructed interface models of the graphene/adenine-M layer or diphenyl ether layer cannot be considered to be identical to the real system for the unknown density of the solution layers, a comparative attempt was still made to obtain better insight into the role of adenine building block to improve the rGO dispersion stability (Fig. S7a and b). The $\mathrm{E}_{\text {int }}$ of the interfacial region between the M NMP solution (adenine-M and diphenyl ether NMP solution) and graphene was calculated as a function of initial distance between layers. As illustrated in Fig. S7c, the $E_{\text {int }}$ of adenine-M system was varied from -132.0910 to $-147.7015 \mathrm{kcal} / \mathrm{mol}$, along with the distance changed from 3 to $9 \AA$. For the comparison system, diphenyl ether, the $E_{\text {int }}$ varied from -114.5288 to -118.6679 $\mathrm{kcal} / \mathrm{mol}$, along with the distance changed from 3 to $9 \AA$. And there were no pronounced trends in the interaction energies as a function of distance between $M$ NMP solution and graphene. It can be easily observed that the $E_{\text {int }}$ of adenine-M system was invariably higher than that of diphenyl ether system, no matter the distance. This indicated the interfacial force between two layers in adenine-M system was stronger than that in diphenyl ether system due to the rigidity and planarity characteristics of the adenine moiety, which may explain the efficiency of $p$-APA in stabilizing $\mathrm{rGO} / \mathrm{GO}$ solution.

As can be found from the MD run of rGO/adenine-M system layer (Fig. S7d), the adenine moiety tended to be stacked face to face with rGO basal plane within 100ps (molecular models were shown in Fig. S8). This result may further aid to understand the non-covalent interaction between nucleobase and ultrathin 2D material.

(a)

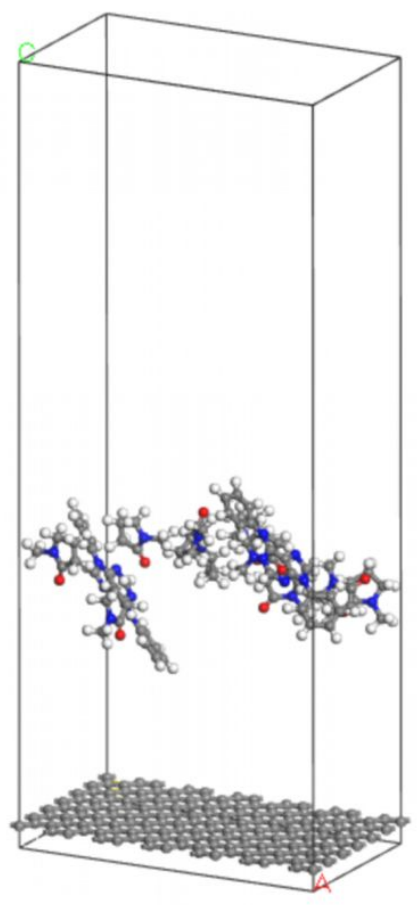

(b)
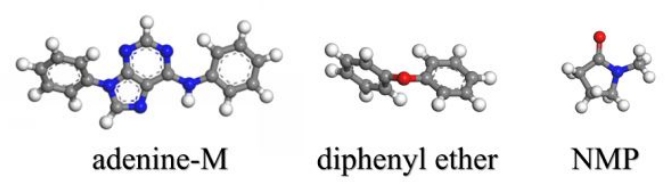

NMP

(c)

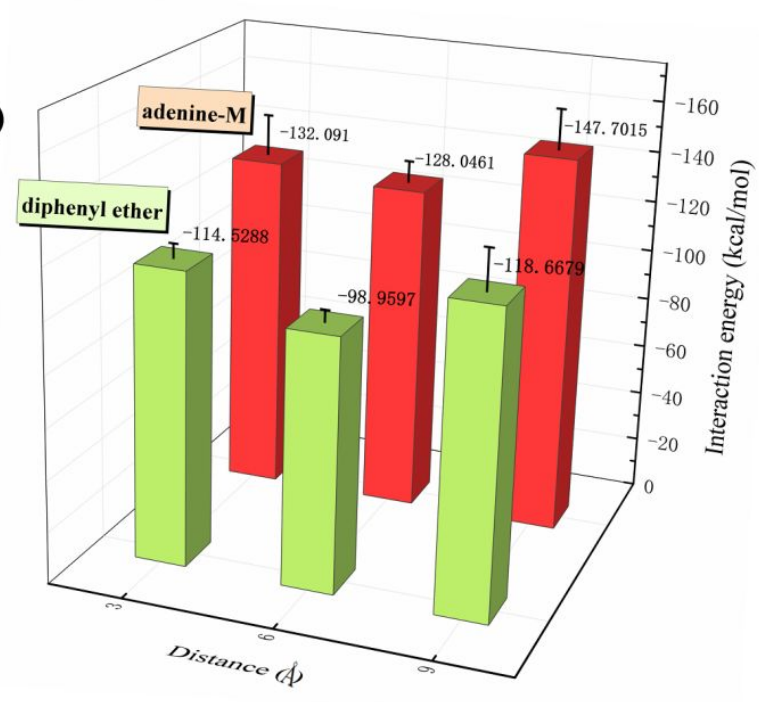




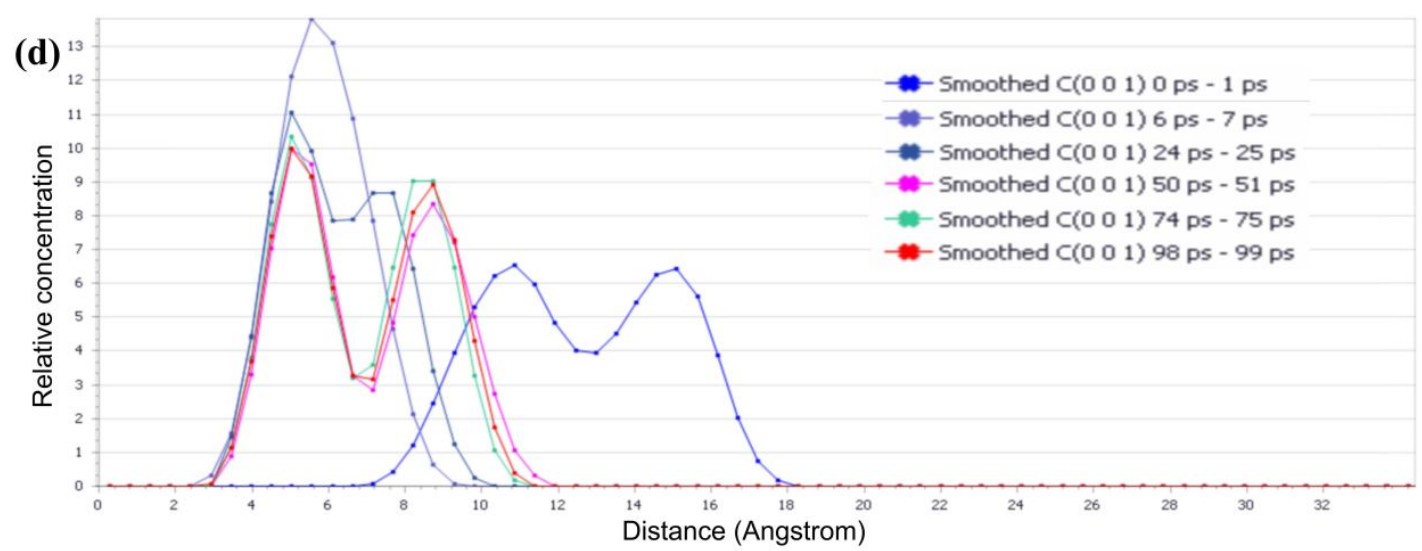

Figure S7. (a) Molecular model of graphene/adenine-M solution in interface region; (b) molecular models of M (adenine-M, diphenyl ether and NMP): stick-ball models, balls with different colors represent different elemental atoms (white for hydrogen, red for oxygen, blue for nitrogen and gray for carbon); (c) $\mathrm{E}_{\text {int }}$ between M NMP solution and graphene in the interface region of mixed solution as a function of the initial distance between $\mathrm{M}-\mathrm{C}$ solution and graphene in the interface region as a function of the initial distance of the layers; (d) MD simulation of rGO/adenine-M system within 100

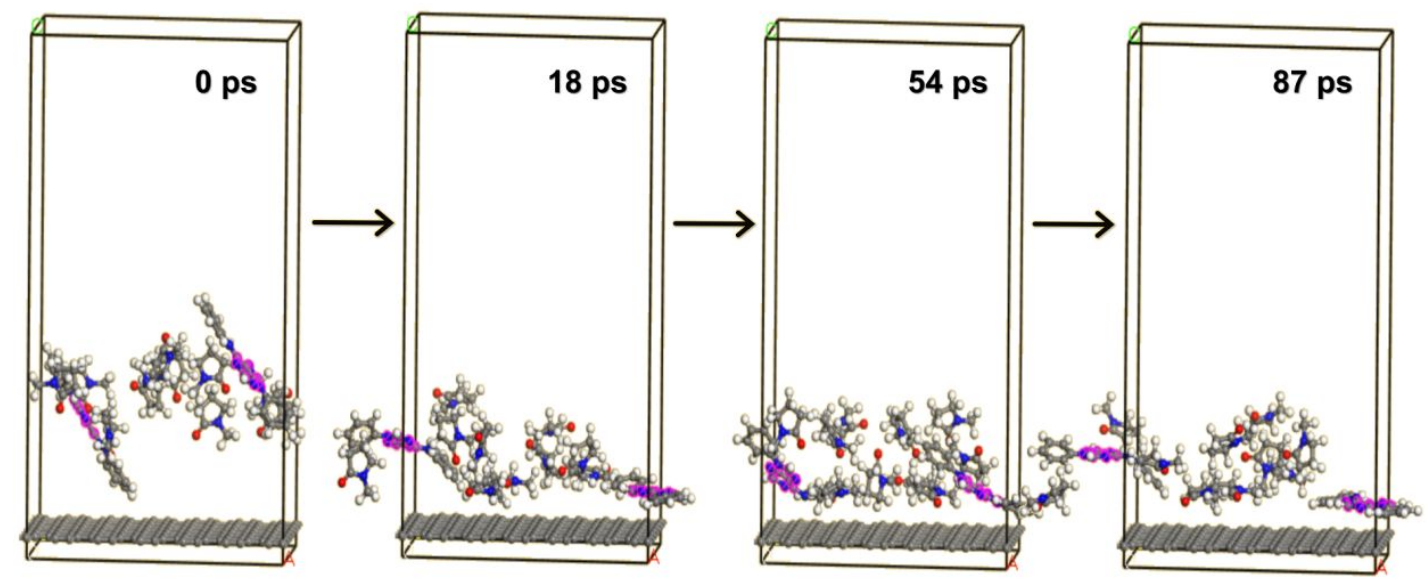

Figure S8. Molecular models of graphene/adenine-M system at different simulation stage (circled molecular represented adenine moiety) 
Table S3. Results of the fitting of the conductivity data in Fig. 12a according to Eq. (3-2)

\begin{tabular}{ccccc}
\hline Content $/ \mathrm{wt} \%$ & \multicolumn{3}{c}{$\mathrm{n}$} \\
\cline { 2 - 5 }$(\text { Vol.\% })^{\mathrm{a}}$ & rGO-API & GO-API & rGO-OPI & GO-OPI \\
\hline $0.5(0.422)$ & 0.12 & 0.14 & 0.15 & 0.19 \\
$1.0(0.593)$ & 0.10 & 0.13 & 0.13 & 0.17 \\
$1.5(0.686)$ & 0.10 & 0.13 & 0.13 & 0.17 \\
$2.0(0.745)$ & 0.10 & 0.12 & 0.13 & 0.16 \\
\hline
\end{tabular}

aThe volume fraction $(\varphi)$ of $\mathrm{rGO} / \mathrm{GO}$ in films was calculated by the following equation:

$$
\varphi=\frac{d_{m} w_{f}}{\left(d_{m}-d_{f}\right) w_{f}+d_{f}}
$$

where $d_{m}$ and $d_{f}$ were the density of the matrix and filler, respectively, and $w_{f}$ was the filling content of filler.

\section{Reference}

[1] S.S. Xantheas, On the importance of the fragment relaxation energy terms in the estimation of the basis set superposition error correction to the intermolecular interaction energy, J. Chem. Phys. 104 (1996) 8821-8824.

[2] H.J. Oh, T. Li, J.Y. An, Drug Release Properties of a Series of Adenine-Based Metal-Organic Frameworks, Chem.-Eur. J. 21 (2015) 17010-17015.

[3] J.E. Freund, M. Edelwirth, P. Krobel, W.M. Heckl, Structure determination of two-dimensional adenine crystals on graphite, Phys. Rev. B 55(1997) 5394-5397.

[4] H. Vovusha, S. Sanyal, B. Sanyal, Interaction of nucleobases and aromatic amino acids with graphene oxide and graphene flakes, J. Phys. Chem. Let. 4 (2013) 3710-3718.

[5] J.H. Hu, R.K. Li, C. Chen, Z. Lu, K. Zeng, G. Yang, New insights into mechanism of negative in-plane CTE based on bio-based adenine-containing polyimide film, Polymer 146 (2018) 133-141.

[6] M.P. Down, S.J. Rowlet-Neale, G.C. Smith, C.E. Banks, Fabrication of Graphene Oxide Supercapacitor Devices, ACS Appl. Energ. Mater. 1 (2018) 707-714.

[7] Z.X. Zhang, H.L. Huang, X.M. Yang, L. Zang, Tailoring electronic properties of graphene by $\pi-\pi$ stacking with aromatic molecules, J. Phys. Chem. Lett. 2 (2011) 2897-2905.

[8] M.J. Deka, D. Chowdhury, Tuning electrical properties of Graphene with different $\pi$-stacking organic molecules, J. Phys. Chem. C 120 (2016) 4121-4129.

[9] Y.Y. Sun, L. Chen, L. Cui, Y.W. Zhang, X.Z. Du, Molecular dynamics simulation of cross-linked epoxy resin and its interaction energy with graphene under two typical force fields, Comp. Mater. Sci. 143 (2018) 240-247. 